\title{
The Weierstrass subgroup of a curve has maximal rank.
}

\author{
Martine Girard, David R. Kohel, and Christophe Ritzenthaler
}

\begin{abstract}
We show that the Weierstrass points of the generic curve of genus $g$ over an algebraically closed field of characteristic 0 generate a group of maximal rank in the Jacobian.
\end{abstract}

The Weierstrass points are a set of distinguished points on curves, which are geometrically intrinsic. In particular, the group these points generate in the Jacobian is a geometric invariant of the curve. A natural question is to determine the structure of this group. For some particular curves with large automorphisms groups (for instance, Fermat curves [18]), these groups have been found to be torsion. The first author provided the first examples where this group has positive rank $([7,8])$ and obtained a lower bound of 11 on the rank of the generic genus 3 curve. The motivation of this paper was to bridge the gap between this bound and the expected bound of $23-$ meaning that there are no relations between the Weierstrass points on the generic genus 3 curve. The result we obtain is valid for generic curves of any genus. More precisely, let the Weierstrass subgroup of a curve $\mathcal{C}$ be the group generated by the Weierstrass points in the Jacobian of the curve $\mathcal{C}$. We show that

Theorem 1. The Weierstrass subgroup of the generic curve of genus $g \geq 3$ is isomorphic to $\mathbb{Z}^{g\left(g^{2}-1\right)-1}$.

As a consequence of this theorem, we deduce the following corollaries.

Corollary 2. For any number field $K$, the group generated by the Weierstrass points of a curve over $K$ in its Jacobian is isomorphic to $\mathbb{Z}^{g\left(g^{2}-1\right)-1}$, outside of a set of curves whose moduli lie in a thin set in $\mathcal{M}_{g}(K)$.

Corollary 3. For each $g \leq 13$, the curves of genus $g$ over $\mathbb{Q}$ for which the group generated by the Weierstrass points in its Jacobian is isomorphic to $\mathbb{Z}^{g\left(g^{2}-1\right)-1}$, determine a Zariski dense set of moduli in $\mathcal{M}_{g}$. 
We start by recalling some basic definitions and properties of Weierstrass points, then some results concerning the behaviour of Weierstrass points under specialisation. We then describe the fundamental tools in our study, which are the natural Galois module structure of the subgroup of divisors with support on the Weierstrass points and the geometric characterisation of the Galois group. Using the specialisation of this Galois module in families, we obtain the main result.

\section{The Weierstrass subgroup of a curve.}

We recall in this section the definition and some properties of Weierstrass points (see [12] exercise A.4.14).

Let $\mathcal{C} / K$ be a smooth projective curve of genus $g \geq 2$ over a field $K$ of characteristic 0 , and let $P$ be any point on $\mathcal{C}$. We say that $P$ is a Weierstrass point if and only if there exists a differential form $\omega \in H^{\circ}\left(C, \Omega_{C}\right)$, such that $\operatorname{ord}_{P}(\omega) \geq g$. Let $\mathcal{W}$ be the set of Weierstrass points on $\mathcal{C}$.

Alternatively we may characterise the Weierstrass points in terms of the dimensions of Riemann-Roch spaces. For any divisor $D$ on $\mathcal{C}$, let $\mathcal{L}(D)$ be the Riemann-Roch space

$$
\left\{f \in K(\mathcal{C})^{*}: \operatorname{div}(f)+D \geq 0\right\} \cup\{0\}
$$

and let $\ell(D)$ be its dimension.

Proposition 4. A point $P$ is Weierstrass if and only if $\ell(g P) \geq 2$.

The gap sequence associated to a point $P$ is defined to be the set

$$
G(P)=\{n \in \mathbb{N}: \ell(n P)=\ell((n-1) P)\} .
$$

We can define the weight of a point to be

$$
w(P)=\left(\sum_{n \in G(P)} n\right)-g(g+1) / 2 .
$$

Positive weight provides yet another characterisation of Weierstrass points and we have a formula for the number of Weierstrass points, counted with multiplicities equal to their weights.

Proposition 5. A point $P$ is a Weierstrass point if and only if $w(P) \geq 1$, and $\sum w(P) P$ belongs to the complete linear system

$$
\left|\frac{g(g+1)}{2} K_{C}\right| \text {. }
$$

In particular, the sum of the weights of all Weierstrass points is $g\left(g^{2}-1\right)$. 
We define the Weierstrass subgroup to be the group generated by the differences of Weierstrass points in the Jacobian of the curve identified with $\operatorname{Pic}^{\circ}(\mathcal{C})$.

\section{Specialisation of Weierstrass points.}

In this section, we recall two theorems on the behaviour of Weierstrass points under specialisation. For a family $\mathcal{C} \rightarrow X$ of smooth projective curves of genus $g$ over an irreducible base $X$. We define $W_{\eta}$ and $W_{s}$ to be the group generated by the Weierstrass points in the generic fibre $\mathcal{C}_{\eta}$ and a special fibre $\mathcal{C}_{s}$, respectively.

Theorem 6. The group of Weierstrass points form an algebraic family such that $W_{\eta}$ surjects on $W_{s}$ and is injective on torsion.

Proof. For the first part, see Hubbard [13] or Laksov-Thorup [15], the second part is classic (see [12] Theorem C.1.4).

We furthermore need the following theorem of Néron which provides constraints on the set of rational points for which the generic rank can decrease under specialisation.

Theorem 7 (Néron [17], see Serre [20], p.152). Let $\pi: \mathcal{A} \rightarrow X$ be a family of abelian varieties over a number field $K$. Then the group $\mathcal{A}(K(X))$ is finitely generated, and the set

$$
\left\{P \in X(K) \mid \mathcal{A}(K(X)) \rightarrow \mathcal{A}_{P}(K) \text { is not injective }\right\}
$$

is a thin set.

N.B This form of the theorem appears in Serre [20] with $X$ any open subvariety of $\mathbb{P}^{n}$, but the proof holds more generally. However, for $X$ of dimension 1 or of general type, the full set $X(K)$ of rational points may be thin for any number field $K$.

\section{The generic Galois group.}

Let $\mathcal{M}_{g}$ be the moduli space of curves of genus $g$, let $\mathcal{C}_{g} \rightarrow \mathcal{M}_{g}$ be the universal curve over $\mathcal{M}_{g}$ (i.e. the moduli space of pointed curves), and let $\pi: \mathcal{W}_{g} \rightarrow \mathcal{M}_{g}$ be the restriction to the locus of Weierstrass points. Eisenbud and Harris 4] studied the geometric monodromy group of this cover, which 
can be identified (see Harris 9]) with the geometric Galois group of $\pi$, i.e. the group of automorphisms of the normal closure of $\mathbb{C}\left(\mathcal{W}_{g}\right) / \mathbb{C}\left(\mathcal{M}_{g}\right)$. They proved that the monodromy group is as large as possible.

Theorem 8 (Eisenbud-Harris [4]). The monodromy group of $\pi$ equals the full symmetric group $S_{g\left(g^{2}-1\right)}$ acting on the $g\left(g^{2}-1\right)$ generic Weierstrass points.

Since $\mathcal{M}_{g}$ can be defined over $\mathbb{Q}$, and the geometric Galois group is maximal, we conclude that the Galois group of the normal closure of $\mathbb{Q}\left(\mathcal{W}_{g}\right) / \mathbb{Q}\left(\mathcal{M}_{g}\right)$ must also be the full symmetric group. We apply this theorem to the Weierstrass subgroup of the Jacobian, as a Galois module, in order to prove Theorem 1 .

\section{Galois module structure.}

Let $\mathcal{C} / K$ be a curve of genus $g$, and let $\mathcal{W}$ be its set of Weierstrass points in $\mathcal{C}(\bar{K})$. Then the absolute Galois group $\mathcal{G}=\operatorname{Gal}(\bar{K} / K)$ acts on the set $\mathcal{W}$. Thus the Weierstrass divisor group,

$$
V=\bigoplus_{P \in \mathfrak{P}} \mathbb{Z} \cdot P
$$

is equipped with a natural $\mathbb{Z}[\mathcal{G}]$-module structure, which acts through $\mathbb{Z}[G]$, where $G$ is the image of $\mathcal{G}$ in $\operatorname{Aut}(\mathcal{W})=S_{g\left(g^{2}-1\right)}$ acting as permutations of $\mathcal{W}$.

Theorem 9. The Weierstrass subgroup of the Jacobian $\mathcal{J a c}\left(\mathcal{C}_{g}\right)$ of the generic curve $\mathcal{C}_{g}$ is either a free group of rank $g\left(g^{2}-1\right)-1$ or a torsion subgroup.

Proof. Both the "degree zero" submodule $V^{\circ}$ of $V$, generated by differences of Weierstrass points, and its submodule $P$ of principal divisors with support in $\mathcal{W}$ are $\mathbb{Z}[\mathcal{G}]$-submodules. From Theorem 8 , we know that $\mathcal{W}$ consists of one orbit of $\mathcal{G}$, which acts through the full symmetric group $S_{n}$, where $n=$ $g\left(g^{2}-1\right)=|\mathcal{W}|$. Thus $P_{\mathbb{Q}}=P \otimes_{\mathbb{Z}} \mathbb{Q}$ is a $\mathbb{Q}\left[S_{n}\right]$-submodule of $V_{\mathbb{Q}}^{\circ}=V^{\circ} \otimes_{\mathbb{Z}} \mathbb{Q}$. Since $V_{\mathbb{Q}}^{\circ}$ is simple as a $\mathbb{Q}\left[S_{n}\right]$-module, it follows that $P_{\mathbb{Q}}$ is either trivial or equal to $V_{\mathbb{Q}}^{\circ}$, and the theorem follows. 


\section{Weierstrass subgroups of cyclic covers of $\mathbb{P}^{1}$.}

In this section, we find curves of any genus $g$ such that the subgroup generated by the difference of two Weierstrass points $P$ and $Q$ has odd order. Comparing this with the Weierstrass subgroup of a hyperelliptic curve, we establish that the generic Weierstrass subgroup can not be a torsion subgroup. First, we state the classical result for the Weierstrass subgroup of a hyperelliptic curve.

Proposition 10. The Weierstrass points of an hyperelliptic curve of genus $g$ generate the 2-torsion subgroup of its Jacobian.

In any genus, there exists cyclic trigonal covers of the projective line. Such covers can be described (see [3] and [14]) by the plane model

$$
C: y^{3}=\prod_{i=1}^{s}\left(x-\alpha_{i}\right) \prod_{j=1}^{t}\left(x-\beta_{j}\right)^{2}
$$

where $\alpha_{i}$ and $\beta_{j}$ are distinct complex numbers and $s$ and $t$ satisfy $s+2 t \equiv$ $0 \bmod 3$ and $t<s$. The genus of $C$ is then equal to $g=s+t-2$.

Proposition 11. There exists a curve of genus $g$ with two Weierstrass points whose difference is a point of order 3 in the Jacobian.

Proof. We take $C$ a trigonal curve as defined above, of genus $g>2$, with $t$ in $\{0,1,2\}$ such that $t \equiv-g+1(\bmod 3)$, and with $s=g-t+2 \geq 2$. Then there exist two nonsingular points $P_{1}=\left(\alpha_{1}, 0\right)$ and $P_{2}=\left(\alpha_{2}, 0\right)$. The functions $f=\left(x-\alpha_{1}\right) /\left(x-\alpha_{2}\right)$ and $1 / f$ are respectively in $\mathcal{L}\left(3 P_{2}\right)$ and in $\mathcal{L}\left(3 P_{1}\right)$, and thus the points $P_{1}, P_{2}$ are Weierstrass points. Moreover, since $\operatorname{div}(f)=3\left(P_{1}-P_{2}\right)$ it follows that $P_{1}-P_{2}$ is a 3 -torsion point in the Weierstrass subgroup of the Jacobian of $C$.

\section{Proof of the main theorem.}

We are now in a position to prove:

Theorem 1. The Weierstrass subgroup of the generic curve of genus $g \geq 3$ is isomorphic to $\mathbb{Z}^{g\left(g^{2}-1\right)-1}$.

Proof. By Theorem 9, the generic Weierstrass subgroup is either a free abelian group or is purely torsion. In the latter case, Theorem [ $]$ implies that 
the generic Weierstrass subgroup is isomorphic with the Weierstrass subgroup of every special curve. We first consider the moduli space $T=\mathcal{M}_{g}^{(m)}$ with $m$-level structure. For $m \geq 3$, the space $T$ is a fine moduli space, with universal cover $\mathcal{D}_{g} \rightarrow T$ such that each fibre $C_{t}$ is a curve of genus $g$ whose isomorphism class determines the moduli point $\pi(t)$ on $\mathcal{M}_{g}$ (see e.g. 6]). On the other hand, the finite covers $\mathcal{D}_{g} \rightarrow \mathcal{C}_{g}$ and $T \rightarrow \mathcal{M}_{g}$ determine a birational morphism of $\mathcal{D}_{g}$ to the fibre product $\mathcal{C}_{g} \times \mathcal{M}_{g} T$, by which we may identify the generic Weierstrass subgroup $W_{\eta}$ with the generic Weierstrass subgroup over $T$ (since then $\mathcal{D}_{g} / \mathbb{Q}(T)$ is isomorphic to $\mathcal{C}_{g} / \mathbb{Q}(T)$ ). By specialising to a hyperelliptic curve $C_{t}$, Proposition 10 implies that, if torsion, the generic Weierstrass subgroup must equal the 2-torsion subgroup. This contradicts the result of Proposition 11] which implies that it must surject on a subgroup of order 3 . We conclude that $W_{\eta}$ contains a point of infinite order, and thus is free of maximal rank.

In the following corollaries we apply the main theorem to the specialisations of the Weierstrass subgroup to a number field $K$. Since the generic Weierstrass subgroup is defined only over the splitting field of $\mathbb{Q}\left(\mathcal{W}_{g}\right) / \mathbb{Q}\left(\mathcal{M}_{g}\right)$, we apply Weil restriction to the generic Jacobian to find a family of abelian varieties over $\mathcal{M}_{g}$ to which we can apply Néron's specialisation theorem. As above, we let $\mathcal{J} a c\left(\mathcal{C}_{g}\right) / \mathbb{Q}\left(\mathcal{M}_{g}\right)$ be the Jacobian of the generic fibre of $\mathcal{C}_{g} \rightarrow \mathcal{M}_{g}$. Set $n=g\left(g^{2}-1\right)$, let $\mathcal{M}_{g}^{\circ}$ be the open subvariety of $\mathcal{M}_{g}$ on which $\mathcal{C}_{g}$ has $n$ distinct Weierstrass points, and let $\mathcal{W}_{g, n}$ be the moduli space of curves with $n$ ordered Weierstrass points, as a finite cover of $\mathcal{M}_{g}^{\circ}$. Now let $A / \mathbb{Q}\left(\mathcal{M}_{g}\right)$ be the Weil restriction of $\mathcal{J} a c\left(\mathcal{C}_{g}\right)$ with respect to the extension $\mathbb{Q}\left(\mathcal{W}_{g, n}\right) / \mathbb{Q}\left(\mathcal{M}_{g}\right)$. Then $A\left(\mathbb{Q}\left(\mathcal{M}_{g}\right)\right)$ is naturally isomorphic to $\operatorname{Jac}\left(\mathcal{C}_{g}\right)\left(\mathbb{Q}\left(\mathcal{W}_{g, n}\right)\right)$, which contains the generic Weierstrass subgroup. By restricting $\mathcal{M}_{g}^{\circ}$ further to an open subvariety, we obtain a family $\mathcal{A} \rightarrow \mathcal{M}_{g}^{\circ}$ with generic fibre $A / \mathbb{Q}\left(\mathcal{M}_{g}\right)$.

Corollary 2. For any number field $K$, the group generated by the Weierstrass points of a curve over $K$ in its Jacobian is isomorphic to $\mathbb{Z}^{g\left(g^{2}-1\right)-1}$, outside of a set of curves whose moduli lie in a thin set in $\mathcal{M}_{g}(K)$.

Proof. We apply Néron's specialisation theorem (Theorem [7) to the family $\mathcal{A} \rightarrow \mathcal{M}_{g}^{\circ}$ above. For every point $P$ in $\mathcal{M}_{g}^{\circ}(K)$ outside of a thin set, the group $\mathcal{A}\left(K\left(\mathcal{M}_{g}\right)\right)=A\left(K\left(\mathcal{M}_{g}\right)\right)$ - and the Weierstrass subgroup in particular specialises injectively to $\mathcal{A}_{P}(K)$. Since $\mathcal{M}_{g}^{\circ}$ is open in $\mathcal{M}_{g}$, the same holds for every $P$ outside of a thin set in $\mathcal{M}_{g}(K)$.

For $g \leq 6$ the moduli space $\mathcal{M}_{g}$ is rational, and the complement of a thin set in $\mathcal{M}_{g}(\mathbb{Q})$ provides a dense set in $\mathcal{M}_{g}$ consisting of moduli of curves 
whose Weierstrass subgroup has maximal rank. More generally, this latter property holds in $\mathcal{M}_{g}(\mathbb{Q})$ for all $g$ up to 13 .

Corollary 3. For each $g \leq 13$, the curves of genus $g$ over $\mathbb{Q}$ for which the group generated by the Weierstrass points in its Jacobian is isomorphic to $\mathbb{Z}^{g\left(g^{2}-1\right)-1}$, determine a Zariski dense set of moduli in $\mathcal{M}_{g}$.

Proof. For each $g \leq 13$ the moduli space $\mathcal{M}_{g}$ is unirational 1, 2, 19, i.e. $\mathcal{M}_{g}$ is covered by a dominant map $\pi: \mathbb{P}^{N} \rightarrow \mathcal{M}_{g}$. Let $\mathcal{A} \rightarrow \mathcal{M}_{g}^{\circ}$ be as above, and let $\mathcal{A}_{U}=\mathcal{A} \times{ }_{\pi} U$ be the base extension to $U=\pi^{-1}\left(\mathcal{M}_{g}^{\circ}\right)$. The Weierstrass subgroup embeds in $\mathcal{A}_{U}\left(\mathbb{Q}\left(\mathbb{P}^{N}\right)\right)=\mathcal{A}\left(\mathbb{Q}\left(\mathbb{P}^{N}\right)\right)$, which specialises injectively to $\left(\mathcal{A}_{U}\right)_{P}(\mathbb{Q})=\mathcal{A}_{\pi(P)}(\mathbb{Q})$ for all $P$ outside of a thin set $Z$ in $U(\mathbb{Q})$. The image $\pi(U(\mathbb{Q})-Z)$ is then a Zariski dense set in $\mathcal{M}_{g}$ consisting of moduli of curves for which the Weierstrass subgroup attains the maximal rank.

Lang conjectures that for a variety $X$ of general type over a number field $F$, for each finite extension $K / F$, the Zariski closure of $X(K)$ is contained in a proper subvariety of $X$ (see [16, pp. 15-20] or [12, §F.5.2]). Since the variety $\mathcal{M}_{g}$ is known to be of general type for $g \geq 24$ (see [5, 10, 11]), Lang's conjecture would imply that for any number field $K$ the set $\mathcal{M}_{g}(K)$ is contained in a proper closed subvariety of $\mathcal{M}_{g}$. Since the complete set of rational points over $K$ is not dense, the density result can not be expected to hold for large $g$. In contrast, the plane curves of degree $d$, determine unirational subvarieties of $\mathcal{M}_{g}$, for $g=(d-1)(d-2) / 2$. An analogous result to Theorem 8 for the Galois group of Weierstrass points of plane curves of degree $d$ (in the spirit of [9]) would be desirable in order to establish the maximality of the rank of the Weierstrass subgroup for plane curves.

Acknowledgements. The authors thank Marc Hindry and René Schoof for interest and comments on an earlier draft of this work.

\section{References}

[1] E. Arbarello E. Sernesi, 'The equation of a plane curve', Duke Math. J. 46 (1979), no. 2, 469-485.

[2] M. C. Chang Z. Ran, 'Unirationality of the moduli spaces of curves of genus 11, 13 (and 12)', Invent. Math. 76 (1984), no. 1, 41-54.

[3] M. Coppens, 'The Weierstrass gap sequences of the total ramification points of trigonal coverings of $\mathbb{P}^{1}$, Ind. Math., 47 (1985), 245-276.

[4] D. Eisenbud J. Harris, 'The monodromy of Weierstrass points', Invent. Math., 90 (1987), 333-341. 
[5] D. Eisenbud J. Harris, 'The Kodaira dimension of the moduli space of curves of genus $\geq 23$, Invent. Math. 90 (1987), no. 2, Invent. Math., 90 (1987), 359-387.

[6] B. van Geemen F. Oort, 'A compactification of a fine moduli space of curves', Resolution of singularities (Obergurgl, 1997), 285-298, Progr. Math., 181 (Birkhäuser, Basel, 2000).

[7] M. Girard, 'Géométrie du groupe des points de Weierstrass d'une quartique lisse', Journal of Number Theory, 94 (2002), 103-135.

[8] M. Girard, 'Groupe des points de Weierstrass sur une famille de quartiques lisses', Acta Arithmetica, 105 (2002), 305-321.

[9] J. Harris, 'Galois groups of enumerative problems', Duke Math. J., 46 (1979), $685-724$.

[10] J. Harris, 'On the Kodaira dimension of the moduli space of curves, II. The even-genus case', Invent. Math., 75 (1984), 437-466.

[11] J. Harris D. Mumford, 'On the Kodaira dimension of the moduli space of curves', Invent. Math., 67 (1982), 23-86.

[12] M. Hindry J. H. Silverman, Diophantine Geometry, An Introduction, Graduate Texts in Mathematics, 201 (Springer-Verlag, New York, 2000).

[13] J. H. Hubbard, 'Sur les sections analytiques de la courbe universelle de Teichmüller', Mem. Amer. Math. Soc. 166 (1976).

[14] T. Kato, 'On Weierstrass point whose first non-gaps are three', J. reine angew. Math, 316 (1980), 99-109.

[15] D. Laksov A. Thorup, 'Weierstrass points and gap sequences for families of curves', Ark. Mat., 32 (1994), 393-422.

[16] S. Lang, Number theory. III. Diophantine geometry, Encyclopaedia of Mathematical Sciences, 60, Springer-Verlag, 1991.

[17] A. Néron, 'Problèmes arithmétiques et géométriques rattachés à la notion de rang d'une courbe algébriques dans un corps', Bull. de la S. M. F., 80 (1952), 101-166.

[18] D. E. Rohrlich, 'Points at infinity on the Fermat curves', Invent. Math., 39 (1977), 95-127.

[19] E. Sernesi, 'Unirationality of the variety of moduli of curves of genus twelve', Ann. Scuola Norm. Sup. Pisa Cl. Sci. (4), 8 (1981), 405-439.

[20] J.-P. Serre, Lectures on the Mordell-Weil theorem, Aspects of Mathematics, Friedr. Vieweg \& Sohn, 1989.

$\begin{array}{ll}\text { Martine Girard, David Kohel } & \text { Christophe Ritzenthaler } \\ \text { School of Mathematics and Statistics Institut de Mathématiques de Luminy } \\ \text { The University of Sydney NSW 2006 } 163 \text { Avenue de Luminy, Case 907 } \\ \text { Australia } & \text { 13288 Marseille Cedex 9 } \\ \text { girard@maths.usyd.edu.au, } & \text { France } \\ \text { kohel@maths.usyd.edu.au } & \text { ritzenth@math.jussieu.fr }\end{array}$

